St udy of Surface Danage caused by Laser I rradi at i on for Quant i t at i ve Hydr ogen Anal ysi s i n Zi rcal oy usi ng Laser - i nduced PI asma Br eakdown Spect romet ry

\begin{tabular}{|l|l|}
\hline 著者 & $\begin{array}{l}\text { FUKUMOTO K, YAMADA N, N K H, MARUYAMA T, } \\
\text { KAGAWA K }\end{array}$ \\
\hline $\begin{array}{l}\mathrm{j} \text { our nal or } \\
\text { publ i cat i on t i t l e }\end{array}$ & Al P Conf er ence Pr oceedi ngs \\
\hline vol une & 1104 \\
\hline page $\mathrm{r}$ ange & $73-78$ \\
\hline year & 2009 \\
\hline URL & $\mathrm{ht} \mathrm{t} \mathrm{p:} \mathrm{//hdl} \mathrm{.} \mathrm{handl} \mathrm{e.} \mathrm{net} \mathrm{/10098/1983}$ \\
\hline
\end{tabular}




\title{
Study of Surface Damage caused by Laser Irradiation for Quantitative Hydrogen Analysis in Zircaloy using Laser-induced Plasma Breakdown Spectrometry
}

\author{
$\underline{\text { K. Fukumoto }}^{a,{ }^{*}}$, N. Yamada ${ }^{a}$, H. Niki ${ }^{a}$, T. Maruyama $^{b}$ and K. Kagawa ${ }^{c}$ \\ ${ }^{a}$ Dept. of Nucl. Power and Energy Safety Eng., Graduate School of Eng., Univ. of Fukui, Japan. \\ ${ }^{b}$ Tokyo Institute of Technology, Japan \\ ${ }^{c}$ Dept. of Phys., Fac. of Edu. And Regional Studies, Univ. of Fukui, Japan
}

\begin{abstract}
The surface damage caused by laser irradiation is studied to investigate the possibility of performing a depth-profile analysis of the hydrogen concentration in zircaloy- 4 alloys using laserinduced plasma breakdown spectrometry. After laser irradiation, a heat-affected zone extending about 3 $\mu \mathrm{m}$ down from the top surface can be seen. The depth of this heat-affected zone is independent of the laser power density in the range $10^{8}$ to $10^{9} \mathrm{~W} / \mathrm{cm}^{2}$. In order to obtain the depth profile of the hydrogen concentration in zircaloy-4 alloys, the power density of laser shots must be greater than $1.3 \times 10^{9} \mathrm{~W} / \mathrm{cm}^{2}$.
\end{abstract}

Keywords: Laser-induced plasma breakdown spectrometry, surface damage by laser ablation, zirconium alloys.

PACS: 52.38.Mf, 61.80.Ba

\section{INTRODUCTION}

The cladding tubes for uranium pellets in a light-water nuclear plant are commonly made of zircaloy. During operation of the reactor, hot water reacts with the zircaloy surface to form zirconium oxide and hydrogen gas, which then penetrates into and accumulates in the zircaloy cladding tubes. The presence of more than a certain amount of hydrogen in a zircaloy tube is known to cause intolerable structural damage, such as hydrogen cracking and stress corrosion cracking. It may sometimes cause a very serious accident in a nuclear power plant, such as leakage of fission products from surface cracks in a fuel assembly caused by hydrogen cracking [1]. Therefore, the amount of hydrogen in zircaloy tubes must be quantitatively analyzed for estimating both the life cycle of a fuel assembly in a nuclear power reactor and the integrity of its structural materials, such as stainless steel for the primary coolant system and pressure vessel.

Laser-induced plasma breakdown spectrometry (LIBS) has recently been studied for the analysis of hydrogen in zircaloy specimens, and it has been shown that the spectral quality of hydrogen emission in a helium atmosphere with a low pressure of about 10 Torr is much better than that in atmospheric ambient air [2]. The result of 
this study on zircaloy specimens demonstrated the possibility of attaining a very sharp emission line from impure hydrogen with a very low background and practical elimination of spectral contamination of the hydrogen emission arising from surface moisture and water vapor in the atmospheric ambient gas. This was achieved by employing an ultra pure ambient of helium gas as well as proper defocusing of the laser irradiation and a large number of repeated precleaning laser shots at the same spot on the sample surface. Further adjustment of the gating time led to a significant reduction of the spectral width and an improvement of the detection sensitivity to 50 ppm by weight (wppm). The hydrogen concentration in the zircaloy and the height of the spectral peak of hydrogen emission in LIBS were apparently correlated.

However, LIBS is a quasi-destructive measurement method because the laser irradiation ablates the material surface and sputters the elements in it. Moreover, a heat-affected zone and a molten zone are formed in the ablated surface after laser irradiation. This will affect the behavior of hydrogen redistribution below the ablated surface.

This study is aimed at clarifying the damage on the surface of zircaloy specimens caused by laser irradiation during a quantitative hydrogen-concentration analysis using LIBS. The extent of the heat-affected zone during repeated laser irradiation and the geometrical change of the surface after laser irradiation have been investigated.

\section{PROCEDURE FOR LIBS}

The basic experimental setup used in this study is sketched in Fig. 1. The special cylindrical chamber can be heated and maintained at a desired constant temperature. The laser (Nd-YAG, Quanta Ray LAB SERIES, 1064 nm, 8 ns, max. energy of $450 \mathrm{~mJ}$ ) was operated in the Q-switching mode at $10 \mathrm{~Hz}$ repetition rate. The laser output energy was fixed at $100 \mathrm{~mJ}$ by means of a set of filters with an estimated shot-to-shot fluctuation of 3\%, while the laser beam that was focused by a lens of 200 $\mathrm{mm}$ focal length through a quartz window could have its focused position varied by moving the lens away above ( - defocusing) or below (+ defocusing) the sample surface.

Pieces of zircaloy-410 $\mathrm{mm}$ x $10 \mathrm{~mm}$ in cross section and $1 \mathrm{~mm}$ thick were individually hydrided in a gaseous charge device. The specimens were annealed in a vacuum in the $\beta$ region at $1000^{\circ} \mathrm{C}$ for $0.5 \mathrm{~h}$ to avoid surface oxidation and then cooled down to 600 to $700^{\circ} \mathrm{C}$ before being charged in a hydrogen atmosphere with a pressure from 1 to $100 \mathrm{~Pa}$ for 3 to $24 \mathrm{~h}$. The original hydrogen concentration was $5 \mathrm{wppm}$. The specimens employed in this study had various concentrations of added hydrogen ( 0 , 1110 and 4300 wppm).

The chamber was evacuated using a vacuum pump to a pressure of 0.001 Torr and subsequently heated up to $200^{\circ} \mathrm{C}$ to remove most of the moisture in it. Highpurity helium gas was then streamed into the chamber continuously.

The plasma radiation was detected by an optical multichannel analyzer (OMA system, Andor I*Star intensified CCD) with a spectral resolution of $0.009 \mathrm{~nm}$ at 500 $\mathrm{nm}$. 
The geometrical change of the surface shape of laser-irradiated zircaloy samples was investigated by a surface-roughness profiler. The compositional analysis of the irradiated surface before and after irradiation was carried out by Auger electron spectroscopy with a JAMP-7810 apparatus at the Wakasa Wan energy research center, Japan. The specimens were cross-cut at the center of the irradiated area and the crosssectional surface polished to observe the heat-affected zone around the irradiated area. Metallographic observations using optical microscopy and laser confocal microscopy were made for these specimens. Transmission electron microscopy foils of the surface area of laser-irradiated specimens were made using a JEM-9310 focused ion beam (FIB) processing instrument operated at $30 \mathrm{kV}$ by Ga${ }^{+}$ions at the Wakasa Wan energy research center.

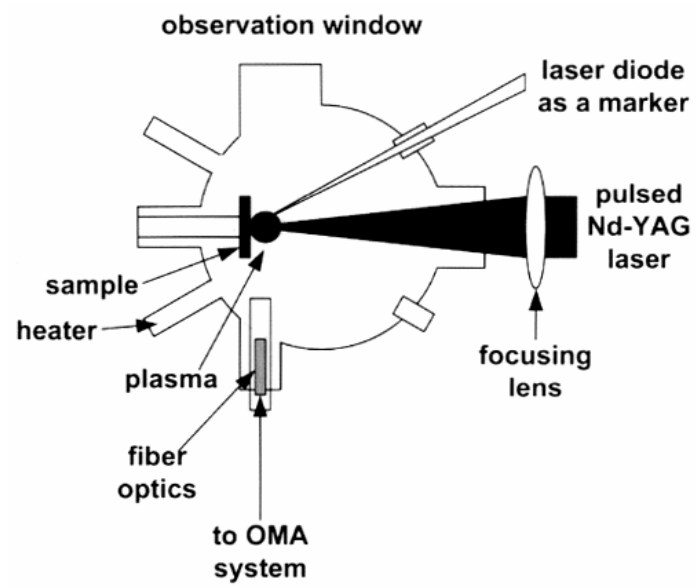

FIGURE 1. A schematic view of the irradiation chamber and experimental setup.

\section{RESULTS}

In order to investigate the effect of impurities on the irradiated surface during laser ablation, a chemical analysis of the laser-ablated surface was performed by Auger electron spectrometry. The atmospheres during laser irradiation were air or helium gas with a low pressure of 10 Torr. The formation of a zirconia oxide film 20 $\mathrm{nm}$ thick was confirmed in the atmospheric air ambient after laser irradiation, but the oxidation film thickness was reduced to $5 \mathrm{~nm}$ in the helium gas with 10 Torr pressure. Therefore helium gas with a low pressure is recommended as the gas atmosphere at the point of impurity incursion during laser irradiation for hydrogen analysis using LIBS.

Defocused laser irradiation is useful for the quantitative analysis of the depth profile of hydrogen concentration in materials, because the laser ablation occurs homogeneously on the irradiated surface area when a flat laser-beam distribution is used. Cross-sectional metallurgical observations of both unirradiated and irradiated areas were made. An irradiated crater was formed by 50 laser shots with $100 \mathrm{~mJ}$ laser energy with $-5 \mathrm{~mm}$ defocusing, corresponding to laser irradiation with $4 \times 10^{9} \mathrm{~W} / \mathrm{cm}^{2}$ power density. A dense dendritic precipitate of zirconium hydride was formed inside the unirradiated specimens. On the other hand, there was no hydride in the heat- 
affected zone extending $3 \mu \mathrm{m}$ down from the top surface in the laser-irradiated area. In the heat-affected zone, there was no fine precipitate of $\mathrm{Zr}(\mathrm{Fe}, \mathrm{Cr})$ intermetallics on the grain boundary close to the $3 \mu \mathrm{m}$ thick surface region from the result of electron probe micro analyzer (EPMA). It is suggested that melting and rapid cooling produce the heat-affected zone $3 \mu \mathrm{m}$ thick in the surface region of zircaloy alloys.

The thickness of the heat-affected zone did not change very much when the number of laser shots increased: it was about 3 to $5 \mu \mathrm{m}$ for specimens laser irradiated with 50, 100 and 300 shots under $-5 \mathrm{~mm}$ defocus conditions, corresponding to a power density of $4 \times 10^{9} \mathrm{~W} / \mathrm{cm}^{2}$. Therefore the number of laser shots does not influence the melting and cooling processes in the heat-affected zone formed by each laser shot. It is suggested that the effects of heat transfer and heat accumulation should be considered to be constant during the laser shots.

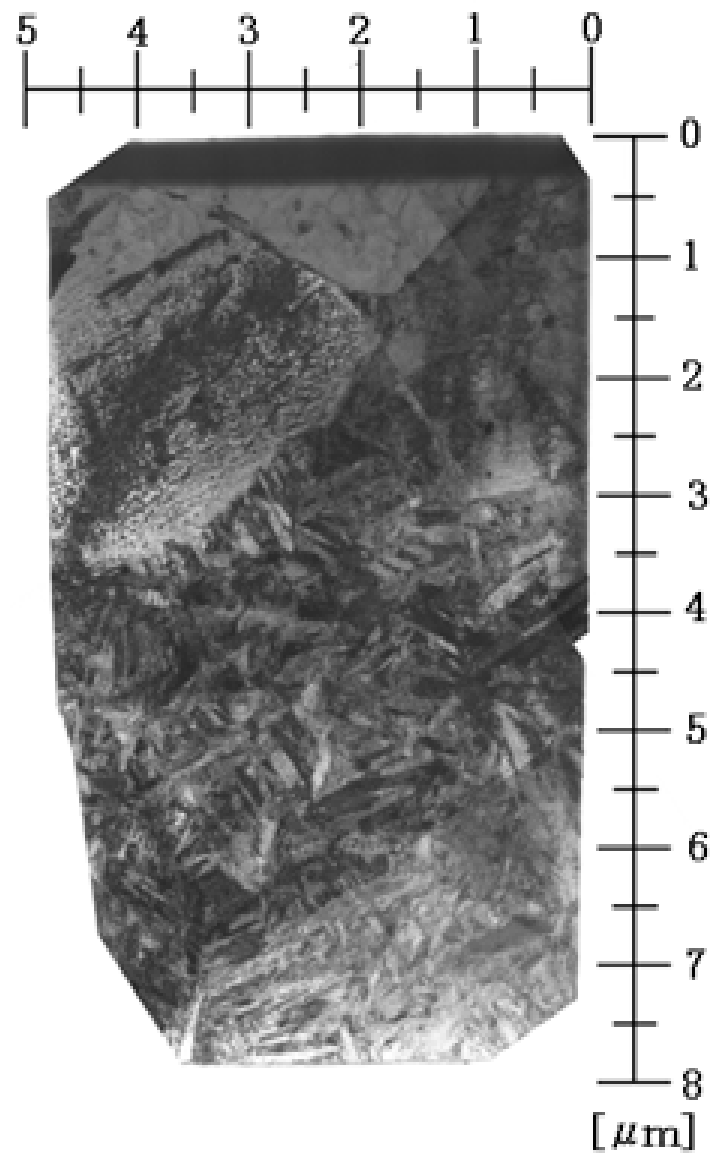

FIGURE 2. A typical TEM photo of cross-sectional FIB-TEM observations on zircaloy-4 alloys containing 1110 wppm of hydrogen. The top surface was irradiated by 100 laser shots with $100 \mathrm{~mJ}$ energy and $-30 \mathrm{~mm}$ defocus conditions.

Fig.2 shows a typical example of cross-sectional FIB-TEM observations of zircaloy-4 alloys containing 1110 wppm of hydrogen. The top surface was irradiated by 100 laser shots with $100 \mathrm{~mJ}$ energy with $-30 \mathrm{~mm}$ defocus conditions, corresponding to a power density of $0.8 \times 10^{9} \mathrm{~W} / \mathrm{cm}^{2}$. The TEM photo shows a crystal region of hexagonal close-packed (hcp) structure, indicating the presence of zirconium crystal grains from the top surface down to a depth of $3 \mu \mathrm{m}$. Below $3 \mu \mathrm{m}$, 
the homogeneous dendritic texture of zirconium hydride can be seen. The hydride was determined by analysis of the electron diffraction pattern [3]. The Debye ring from the diffraction pattern shows that $\varepsilon$-hydride and hcp-zirconium are dominant in the texture. A boundary between the plain grains of zirconium near surface and the grains of dendritic hydride can be clearly seen.

The microstructural analysis shows that the phase transformation from hydride to the original hcp structure occurred within a depth of $3 \mu \mathrm{m}$. It is considered that the hcp crystal region was the heat-affected zone formed by laser ablation. Rapid cooling rate after the melting and dissolution of hydrogen from the hydride make the zirconium hcp phase solidify on the surface area. Compared with the result of metallographic observations for the heat-affected zone irradiated with $-5 \mathrm{~mm}$ defocus conditions, corresponding to $4 \times 10^{9} \mathrm{~W} / \mathrm{cm}^{2}$ power density, the depth profile of the heat-affected zone is not very different from one with $-30 \mathrm{~mm}$ defocus conditions, corresponding to $0.8 \times 10^{9} \mathrm{~W} / \mathrm{cm}^{2}$ power density. It is not clear why the depth of the heat-affected zone does not depend on the power density of the laser. It is considered that most of the incident laser energy is consumed by the ablation of the zircaloy alloy matrix and the amount of energy transferred into the matrix does not change with the incident laser energy. The depth of the heat-affected zone is limited to about 3 to $5 \mu \mathrm{m}$ for the laser-shot process.

The surface roughness of zircaloy-4 alloy was measured before and after laser irradiation as a function of the number of laser shots and the defocus conditions. Fig. 3 shows the change of surface shape, in terms of the mean height and mean depth of craters, obtained from the surface-roughness measurements. With an increasing number of laser shots, the height and depth of craters increase. In the case of tightfocus irradiation conditions, corresponding to $6 \times 10^{9} \mathrm{~W} / \mathrm{cm}^{2}$ power density, a linear relationship between the number of laser shots and the height and depth of craters was obtained. When the defocus condition was $-20 \mathrm{~mm}$, corresponding to $1.3 \times 10^{9} \mathrm{~W} / \mathrm{m}^{2}$ power density, the slope of the linear relationship between the number of laser shots and the height and depth of craters is quite small and the slope is almost zero for -40 $\mathrm{mm}$ defocus conditions, corresponding to a power density of $0.5 \times 10^{9} \mathrm{~W} / \mathrm{m}^{2}$. In the case of a power density less than $1 \times 10^{9} \mathrm{~W} / \mathrm{m}^{2}$, ablation is no longer the dominant process for consuming the laser energy and melting is the dominant process for surface modification. In order to analyze the depth profile of the hydrogen concentration, the power density of the laser shots must be more than $1.3 \times 10^{9} \mathrm{~W} / \mathrm{m}^{2}$. Considering with the result of surface damage, it is suggested that more than $3 \mu \mathrm{m}$ of depth for each shot is required not to influence the heat effect for hydrogen redistribution after laser ablation.

\section{SUMMARY}

In order to perform quantitative analyses of the hydrogen concentration and its depth profile, the surface damage due to laser ablation in a LIBS experiment is investigated. Helium gas with a low pressure is recommended as the gas atmosphere at the point of impurity incursion during laser irradiation for hydrogen analysis by LIBS. The depth of the heat-affected zone formed by laser irradiation is about 3 to $5 \mu \mathrm{m}$, and 
it is likely to be independent of the power density and laser-shot sequence. In order to determine the depth profile of the hydrogen concentration, the power density of the laser shots must be more than $1.3 \times 10^{9} \mathrm{~W} / \mathrm{m}^{2}$. More than $3 \mu \mathrm{m}$ of depth for each shot is required not to influence the heat effect for hydrogen redistribution after laser ablation.

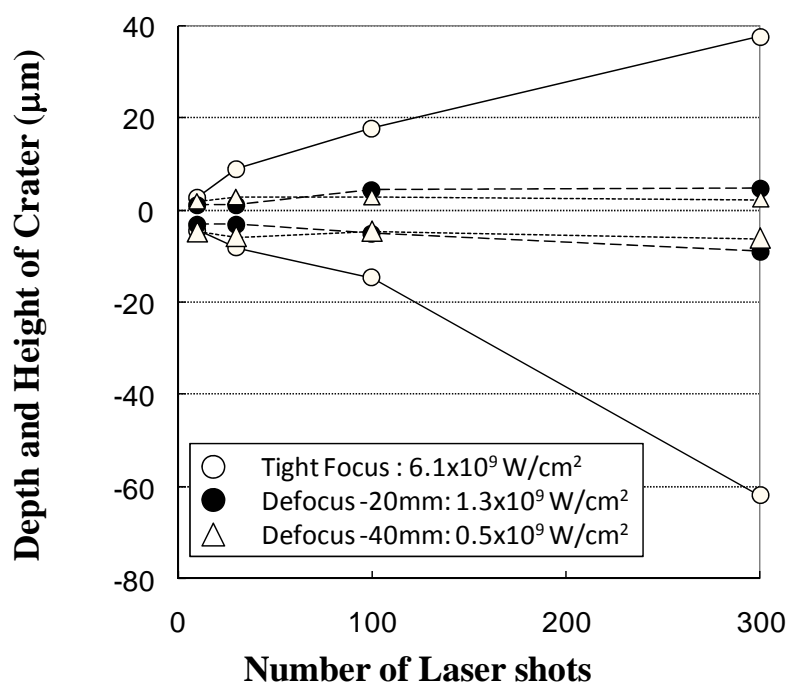

FIGURE 3. Dependence of the mean depth and mean height of laser-irradiation craters in a zircaloy-4 alloy on the number of laser shots.

\section{ACKNOWLEDGMENTS}

This study includes the results of "R\&D for on-site technology of hydrogen analysis in nuclear materials using laser light” entrusted to the University of Fukui by the Ministry of Education, Culture, Sports, Science and Technology of Japan (MEXT).

\section{REFERENCES}

1. J. T. A. Roberts, "Structural Materials in Nuclear Power Systems", Plenum Publishing Co., New York, 1981, pp. 1-51.

2. M. Ramli, K. Fukumoto, H. Niki, S. N. Abdulmadjid, T. Maruyama, K, Kagawa, "Quantitative hydrogen analysis of zircaloy-4 in laser-induced breakdown spectroscopy with ambient helium gas", Applied Optics, 46, pp.8298-8304 (2007)

3. S.Mishra, K.S.Sivaramakrishnan, and M.K.Asundi: J. Nucl. Mater., 45, pp.235-244 (1972-73) 